\title{
Coupling of an Electrical Arc Model With FEM for Vacuum Interrupter Designs
}

\author{
Julien Fontchastagner ${ }^{1}$, Olivier Chadebec ${ }^{2}$, Hans Schellekens ${ }^{1}$, Gérard Meunier $^{2}$, and Vincent Mazauric ${ }^{1}$ \\ ${ }^{1}$ Schneider Electric, 38050 Grenoble cedex 9, France \\ ${ }^{2}$ Laboratoire d'Electrotechnique de Grenoble (LEG_-INPG/UJF/CNRS)—LEG/ENSIEG, \\ UMR INPG/CNRS 5529, 38402 St. Grenoble Cedex, France
}

\begin{abstract}
We present a model of a rotating arc by coupling a finite element method (FEM) and an arc model. A FEM is used to calculate magnetic field between electrodes taking into account the real current distribution in the contacts and in the arc; moreover, ferromagnetic effects and induced currents can be taken into account. A phenomenological arc model is used to predict the arc voltage, which depends on the local magnetic field and the arc length. This arc voltage is updated as the arc displaces itself across the contact surface. The information about arc voltage and local circuit equations is sufficient to find the velocity of the moving arc; hence this model seems more effective than models using Lorentz-forces to describe arc movement which need $a$ priori knowledge about viscosity. This presented method seems to be a promising tool to describe the behavior of rotating arcs in vacuum circuit breakers.
\end{abstract}

Index Terms-Electrical arc model, finite element method (FEM), vacuum arc, vaccum circuit breakers.

\section{INTRODUCTION}

$\mathbf{T}$ HE goal of circuit breakers is to switch off currents in electric circuits. During this process, the contact opening ignites an electrical arc between two electrodes which has to be extinguished as quickly as possible. In this paper, we focus on medium voltage vacuum circuit breakers $(24 \mathrm{kV})$. The vacuum interrupters technology is now well known to be more efficient and cost less than traditional SF6 technology. However, behavior of electrical arc in vacuum is complex and still not really understood.

The magnetic field created by the current flowing inside the electrodes and the arc itself strongly influences the arc behavior by yielding its motion (Laplace's force). Two kinds of geometry are developed by interrupters manufacturers which are associated to two different electrodes geometry and so to two different magnetic field directions. The first one is called axial magnetic field (AMF). In this technology, the magnetic field is axial and can be created by an external coil where a current flows. The arc then gets a diffuse mode for a sufficient value of the field and is then easy to extinguish. The second technology is called radial magnetic field (RMF). In such devices, the magnetic field is radial and created by specific electrodes geometry. The Laplace's force acting on arc is then orthoradial, the arc remains in a concentrate mode which can be represented by simple lines and displaces itself across the contact surface with a rotation motion (see Fig. 1).

In this paper, we focus on RMF interrupters which are known to be less expensive than AMF, with equivalent efficiencies. The development of new electrodes geometry requires adapted modeling tools, first to best understand the arc behavior and afterwards to improve the interrupters design process by reducing the number of mock-up.

Digital Object Identifier 10.1109/TMAG.2005.845023

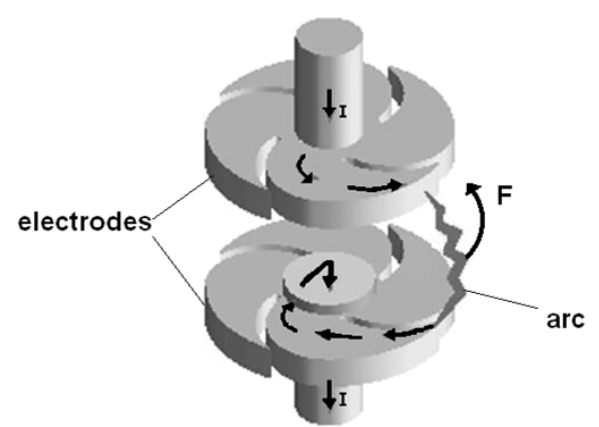

Fig. 1. RMF vacuum circuit breaker with electrodes, electrical arc and force acting on it.

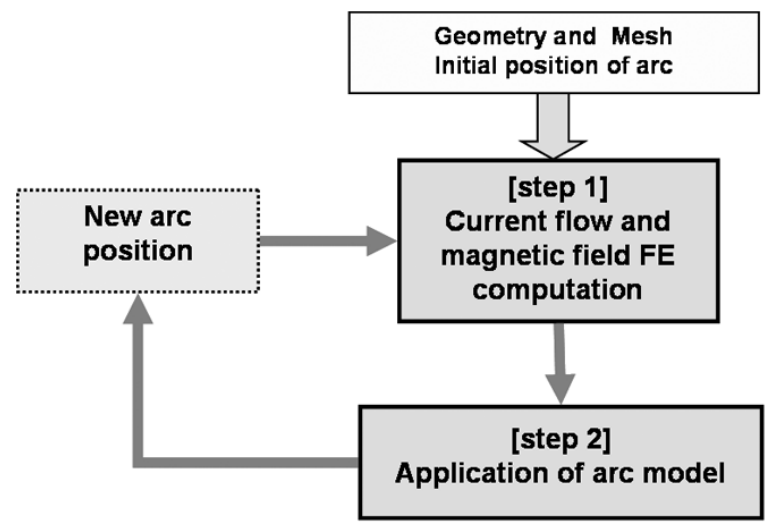

Fig. 2. Coupling principle of the developed tool.

This work proposes an original approach which couples a simple arc model with a FEs magnetic field computation. The approach can be summarized by Fig. 2 .

First, electrodes and air region between and around them are meshed and an initial arc position is chosen. With an adapted FEs formulation, the current flowing in the electrodes and the resulting magnetic field are computed (step 1). Once the magnetic field distribution is obtained, an arc model is applied and leads to a new arc position (step 2). A new field distribution is 
then calculated and so on. Then, we obtained a tool that predicts arc displacement for a given geometry of electrodes. The second part of this paper will explain the FE formulation which allows to compute the magnetic field. The third part focuses on electrical arc model. The last one will explain an approach to calculate arc velocity.

\section{MAGNetic Field COMPUTATION}

Classical approaches for circuit breakers modeling are based on the computation of the magnetic vector potential A [1], [2]. In these works, the arc and the electrodes are meshed. The first step is to calculate the current density in both arc and electrodes by a finite element (FEM) and then a second FE resolution leads to $\mathbf{A}$. The field can then easily be deduced from $\mathbf{A}$ and the force is calculated thanks to the integration of Laplace's force on the arc region. The drawback of this approach is that if we want to apply an arc model to make the arc move it is necessary to create a new mesh of the air and the arc region at each step. This method is then time-consuming and difficult to implement.

In our approach, we preferred to use the $\mathbf{T}_{\mathbf{0}}-\boldsymbol{\Phi}$ formulation which can easily take into account conductors modeling by inductors. In particular, we will see that by modeling our arc with an inductor, no new mesh at each time step is required.

This chapter is divided in three different parts. First, the distribution of current is calculated, then the field created by these current is computed, finally the coupling with circuit equation is done and the reaction of ferromagnetic part is computed.

\section{A. Computation of Current Distributions}

The current distribution $\mathbf{J}_{\mathbf{0 i}}$ in each electrodes $i$ is calculated for 1 A by a static current flow FEM. The electrical potential $V$ is used and the equation to be solved is

$$
\operatorname{div}(\sigma \operatorname{grad} V)=0
$$

where $\sigma$ is the electrical conductivity of electrodes. For each electrode, the potential on the input and on the arc contact point is imposed. The electric field is assumed to be tangential everywhere. Once the FEs problem is solved, a distribution of $V$ is obtained and $\mathbf{J}_{\mathbf{0} \mathbf{i}}$ can be calculated by

$$
\mathbf{J}_{\mathbf{0 i}}=-\sigma \operatorname{grad} V \text {. }
$$

\section{B. Computation of the Magnetic Field}

We need now to compute the magnetic field created by both electrodes and the arc itself. Two different methods are used.

1) Magnetic Field $\boldsymbol{H}_{\mathbf{O}_{\text {arc }}}$ Created by Arc: We consider that the arc position is known. It is represented by a broken line carrying currents of $1 \mathrm{~A}$. This representation of currents is wellknown in electromagnetism modeling and is called inductor. The magnetic field created by such distribution can easily be computed with an analytical formula. According to law of BiotSavart, this field $\boldsymbol{H}_{\mathbf{O}_{\text {arc }}}$ is equal to

$$
\mathbf{H}_{0_{\text {arc }}}=\frac{1}{4 \pi} \int_{\text {arc }} \frac{\mathbf{u} \times \mathbf{r}}{\mathbf{r}^{3}} d V
$$

where $\mathbf{u}$ is a vector tangential to the arc at the integration point and $\mathbf{r}$ is the vector linking the integration point to the point where the field is computed.

2) Magnetic Field $T_{\text {elec }}$ Created by Electrodes: The magnetic field $\mathbf{H}_{0 \mathbf{i}}$ created by each electrode $\mathrm{i}$ can also be calculated by law of Biot-Savart. However this approach is very time consuming. A better way is to consider a magnetostatic FEM, by solving Maxwell-Ampere's equation

$$
\left(\boldsymbol{r o t} \mathbf{H}_{\mathbf{0 i}}-\mathbf{J}_{\mathbf{0 i}}\right)^{2}=0 .
$$

3) Main Finite Element Resolution: On the one hand, if there is no ferromagnetic material in the devices, the magnetic field in the area between electrodes is the sum of the two previous fields

$$
\mathbf{H}=\left(\mathbf{H}_{\mathbf{0}_{\text {arc }}}+\sum_{i=1}^{2} \mathbf{H}_{\mathbf{0 i}}\right) I
$$

where $\mathbf{I}$ is the current flowing to the circuit breaker. On the other hand, if circuit breaker contains ferromagnetic materials, the field becomes

$$
\mathbf{H}=\left(\mathbf{H}_{\mathbf{0}_{\text {arc }}}+\sum_{i=1}^{2} \mathbf{H}_{\mathbf{0 i}}\right) I-\operatorname{grad} \Phi
$$

where $-\operatorname{grad} \Phi$ represents the field created by the ferromagnetic part. $\Phi$ is obtained by the following FE's resolution (free divergence of the induction)

$$
\operatorname{div}\left(\mu\left(\mathbf{H}_{\mathbf{0}_{\text {arc }}}+\sum_{i=1}^{2} \mathbf{H}_{\mathbf{0 i}}\right) I-\operatorname{grad} \Phi\right)=0
$$

coupled with circuit equations [3]. $\mu$ is then the magnetic permeability of the material.

As we saw, several FE's resolution are needed (three without any ferromagnetic material and four with). However, the main advantage of this approach is that the arc position does not depend on the mesh (the magnetic field created by the arc is represented by an inductor). In particular, no new mesh at each step is required to take into account the motion.

\section{Coupling With ARC Model}

The diffuse arc model which is outlined in [4], is implemented with the following improvement. Let us notice that the following model has been developed by taking into account experimental observations.

- An electrical arc aligns itself with the respect to the magnetic lines.

- On each node located at the neighborhood of the arc contact node of the bottom electrode, we calculate each possible path. Paths which are loops shaped or which do not reach the top electrode are eliminated.

- The new arc position is on the path with lowest arc voltage. For each possible arc's position, an arc voltage can be calculated thanks to the Gundlach's formula [4]

$$
U_{i}=\sqrt{B} \log \left(\frac{d}{d_{0}}\right)
$$




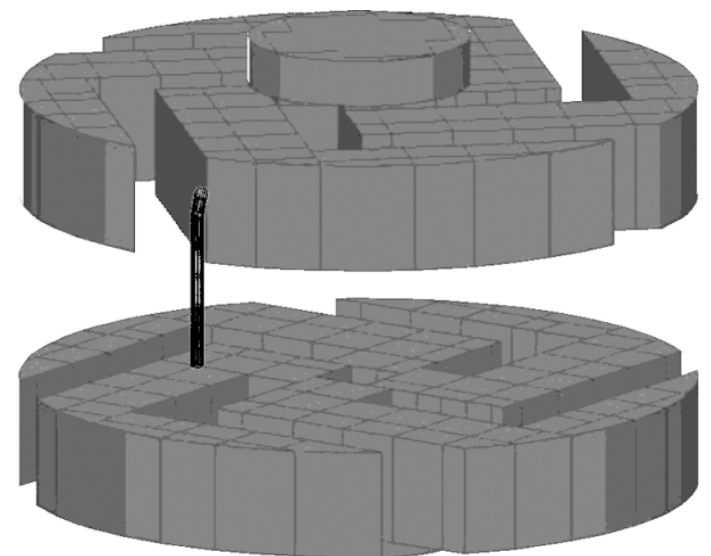

Fig. 3. Arc position (second step of computation). The arc is close to its initial position.

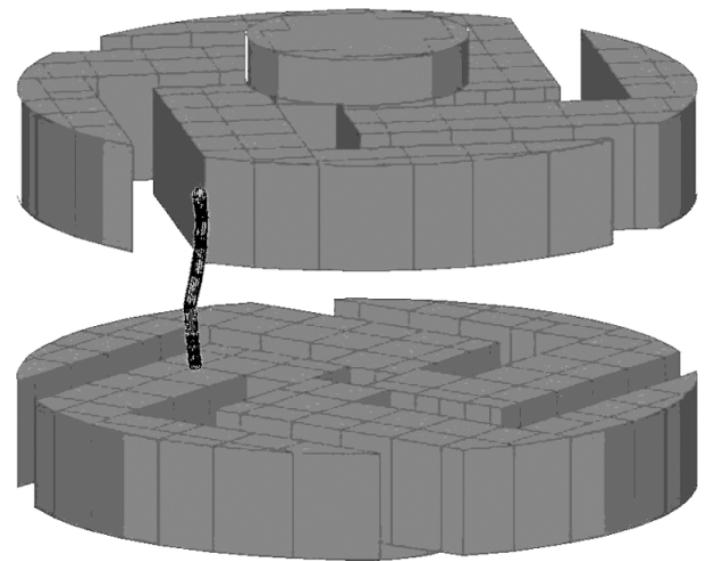

Fig. 4. Arc position (fourth step of computation). The arc begins to get out of its initial shape.

where $d$ is the length of the arc and $d_{0}$ the distance between electrodes.

A new arc position is then found. Inductors which represent the arc are displaced and electrical potential conditions on the contact are changed. A new magnetic field is computed and so on.

\section{NUMERICAL RESULTS}

The model arc has been implemented in FLUX3D software. It has been tested on a realistic geometry of RMF circuit breaker without any ferromagnetic parts $(\Phi=0)$. Figs. 3-6 show the arc behavior obtained. As we see, the arc starts from the center of the electrodes and reach their boundaries. This motion is due to the radial magnetic field.

\section{TOWARD A TIME-TEPING APPROACH}

The following approach does not take into account the velocity of arc. In particular, the time is not computed between to arc position. An estimation of this time could allow to predict the velocity and moreover to estimate the influence of eddy currents which decrease the magnetic field and then influence the current interruption process. This section presents preliminary considerations to include time in our model.

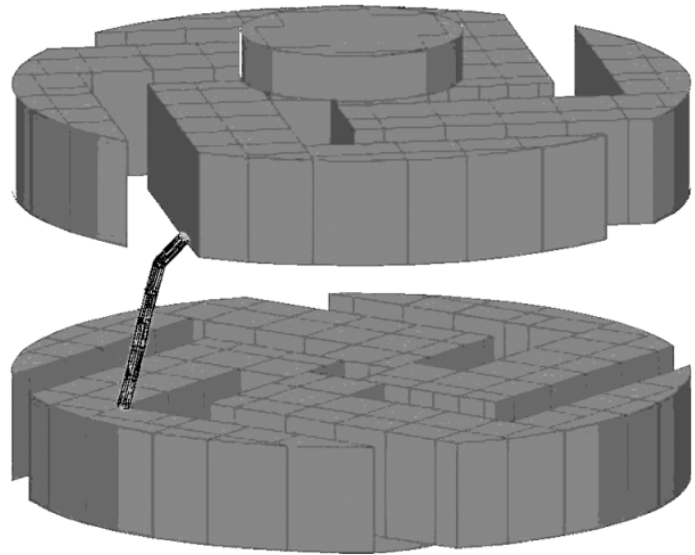

Fig. 5. Arc position (sixth step of computation). The arc begins to move from the center of the electrode to its circumference.

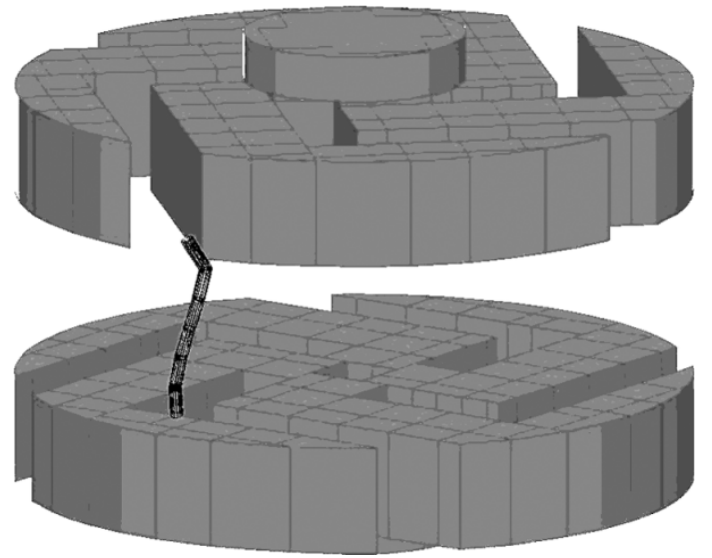

Fig. 6. Arc position (eighth step of computation). Beginning of the rotation motion.

\section{A. Equivalent Circuit Representation}

Our approach is based upon several approximations. The time between two successive arc positions will be called $\Delta t$. It is assumed that the two arcs coexist during this period. Moreover, the two arcs voltages are assumed to remain constant and to be equal to $U_{0}$ and $U_{0}+\Delta U$ respectively. At $t=t_{0}$, a current $I c$ flows into the first arc (Xo position), the currents flowing into the second one is equal to 0 . At $t=t_{0}+\Delta t, I c$ flows into the second one, the current in the first arc is zero. We can then represent the change of position between two arcs by a current $i b$ flowing in a loop composed by both electrodes and both arcs (see Fig. 7).

We can then model the phenomena by a simple circuit representation (see Fig. 8).

$R$ represents the sum of the resistances between the contact points of the two arcs. It can be easily computed by a classical current flow FEM. $L$ is the inductance of the circuit composed by arcs and the path of currents $i b$ in electrodes. By approaching the current path by simple lines, the $L$ value can be roughly estimated with analytical formula [5]. $\Delta U$ is the difference between the two arc voltages computed with (8). The expression of $i b$ are obtained by solving a classical $R L$ circuit differential equation

$$
i b(t)=\frac{-\Delta U}{R} \cdot\left(1-e^{-(R / L) t}\right) .
$$




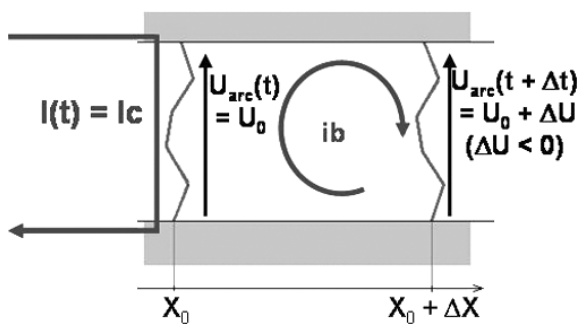

Fig. 7. Representation of two successive arc positions and of the current flowing between them.

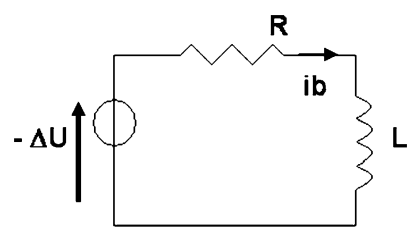

Fig. 8. Equivalent circuit representation.

Let's consider the critical resistance $R_{c}$ expressed by

$$
R_{c}=-\frac{\Delta U}{I c} .
$$

If this resistance $R_{c}$ is greater than $R$, then the current $i b$ cannot reach $I c$ value and the arc will not move. Then a new admissible with a higher voltage arc is tested (see Section III). When no more admissible arc is available, the arc is considered as static and no motion is possible. If $R_{c}$ is smaller than $R, i b$ reaches $I c$ in a finite time and the arc moves effectively to the second position. The time $\Delta t$ can then be evaluated with the following expression:

$$
\Delta t=-\frac{L}{R} \cdot \ln \left(1+\frac{R \cdot I c}{\Delta U}\right) .
$$

The momentary velocity of $\operatorname{arc} V$ can be obtained by

$$
V=\frac{\Delta x}{\Delta t} .
$$

\section{B. Eddy Currents Computation}

Moving conductors like the arcs create flux variation in massive conductors (electrodes), which leads to eddy current in these electrodes. These eddy currents decrease the magnetic field. Then, it could be useful to compute them.

For this, it is then necessary to develop a Lagrangian description, where each moving conductor has its own coordinate system. A step by step resolution is adopted and, at each time step, the following equations are solved by a finite difference time-stepping approach with an implicit scheme [6], [7]

$$
\begin{aligned}
\operatorname{curl}(\rho \operatorname{curl} \mathbf{H}) & =-\frac{d(\mu \mathbf{H})}{d t} \\
\operatorname{div}(\mu \mathbf{H}) & =0
\end{aligned}
$$

where $\rho$ is the resistivity of the material, $\mu$ is the permeability, and $\mathbf{H}$ is the magnetic field. $d / d t$ denotes the convective derivative. In the finite difference approach $\Delta t$ is computed by (11). This approach has not been implemented yet in the final tool but has already shown its efficiency in [7].

\section{CONCLUSION}

We have developed a new tool to predict arcs behavior in vacuum circuit breakers. This approach is based upon the coupling between powerful magnetic field FE computation and a simple rotating arc model. The method leads to quite good results by showing an arc behavior in agreement with the experimental observations. This model seems more effective than models using Lorentz forces to describe arc movement which need a priori knowledge about viscosity and mass of the arc. This here presented method seems to be a promising tool to describe the behavior of rotating arcs in vacuum circuit breakers and should help interrupters designers to develop new geometry. Future results will deals with the comparison between arc velocity obtained by this model and the experiments.

\section{ACKNOWLEDGMENT}

This work was supported in part by Schneider Electric.

\section{REFERENCES}

[1] A. Heno, T. Altimani, P. Picot, and H. Schellekens, "3D finite element simulation and synthetic tests of vacuum interrupters with axial magnetic field contacts," in Proc. 20th Int. Symp. Discharges and Electrical Insulation in Vacuum, 2002, pp. 463-466.

[2] Y. Kawase, H. Mori, H. Inoue, and S. Ito, "3-D finite element analysis of magnetic blowout forces acting on the arc in vacuum circuit breakers," IEEE Trans. Magn., vol. 32, no. 3, pp. 1681-1684, May 1996.

[3] Y. LeFloch, G. Meunier, C. Guerin, P. Labie, X. Brunotte, and D. Boudaud, "Coupled problem computation of 3-D multiply connected magnetic circuits and electric circuits," IEEE Trans. Magn., vol. 39, no. 3, pp. 1725-1728, May 2003.

[4] H. Schellekens, "Arc behavior in axial magnetic field vacuum interrupters equipped with an external coil," presented at the 18th Int. Conf. Discharges and Electrical Insulation, Eindhoven, The Netherlands, Aug. 1998.

[5] L. Landau and E. Lifschitz, Classical Theory of Fields. Moscow, U.S.S.R.: Mir, 1970, vol. 2, Course of Theoretical Physics.

[6] G. Meunier, Y. Le Floch, and C. Guérin, "A nonlinear coupled circuit $t-t_{0}-F$ formulation for solid conductors," IEEE Trans. Magn., vol. 39, no. 3, pp. 1729-1732, May 2003.

[7] O. Chadebec, G. Meunier, V. Mazauric, and Y. Le Floch, "Eddy currents effects in circuit breakers during arc displacement phase," IEEE Trans. Magn., vol. 40, no. 2, pp. 1358-1361, Mar. 2004.

Manuscript received June 8, 2004. 